\title{
Rectotumoral fistula formation occurring more than 5 years after carbon ion radiotherapy for sacral chordoma: A case report
}

\author{
YUICHIRO UKON ${ }^{1,2}$, HIDETATSU OUTANI $^{1,2}$, SHIGENORI NAGATA $^{3}$, EIICHI KONISHI ${ }^{4}$, REIKO IMAI ${ }^{5}$, \\ YUSUKE DEMIZU $^{6,7}$, TOMOAKI OKIMOTO ${ }^{7}$, NORIFUMI NAKA ${ }^{2}$ and NOBUHITO ARAKI ${ }^{2,8}$ \\ ${ }^{1}$ Department of Orthopaedic Surgery, Osaka University Graduate School of Medicine, Suita, Osaka 565-0871; \\ ${ }^{2}$ Musculoskeletal Oncology Service, Osaka International Cancer Institute, Osaka, Osaka 541-8567; \\ ${ }^{3}$ Department of Diagnostic Pathology and Cytology, Osaka International Cancer Institute, Osaka, Osaka 541-8567; \\ ${ }^{4}$ Department of Surgical Pathology, Kyoto Prefectural University of Medicine, Kamigyo-ku, Kyoto 602-8566; \\ ${ }^{5}$ Research Center Hospital for Charged Particle Therapy, National Institute of Radiological Sciences, Chiba, \\ Chiba 263-8555; ${ }^{6}$ Department of Radiation Oncology, Hyogo Ion Beam Medical Center Kobe Proton Center, \\ Kobe, Hyogo 650-0047; ${ }^{7}$ Department of Radiology, Hyogo Ion Beam Medical Center, Tatsuno, Hyogo 679-5165; \\ ${ }^{8}$ Department of Orthopaedic Surgery, Ashiya Municipal Hospital, Ashiya, Hyogo 659-8502, Japan
}

Received October 22, 2018; Accepted February 27, 2019

DOI: $10.3892 / \mathrm{mco} .2019 .1821$

\begin{abstract}
Chordoma is a rare tumor that originates from the notochord. Half of chordomas involve the sacral region. Surgery is considered to be the standard treatment for sacral chordoma. However, carbon ion radiotherapy (CIRT) has recently emerged as a promising treatment for unresectable sacral chordoma. Little is known about the long-term complications of CIRT. We present two cases of rectotumoral fistula formation that occurred $>5$ years after CIRT for sacral chordoma. We considered two possible explanations for fistula formation: radiation enterocolitis after CIRT might cause formation of the fistula long-term, and tumor regrowth might compress the rectum and cause fistula formation. A biopsy in Case 1 showed that regrowth tumor was post-CIRT. It is important to be aware of the possibility of rectal complications after CIRT, and if found, resection of the rectum should be considered. This is a first report of rectotumoral fistula formation that occurred $>5$ years after CIRT for sacral chordoma.
\end{abstract}

\section{Introduction}

Chordoma is a rare tumor that originates from the notochord, with an incidence of $<0.1 / 100,000$ (1). Half of chordomas involve the sacral region, followed by the skull base $(35 \%)$ and the vertebral column (15\%) (2). Surgery is still considered to be

Correspondence to: Dr Nobuhito Araki,Department of Orthopaedic Surgery, Ashiya Municipal Hospital, 39-1 Asahigaoka-cho, Ashiya, Hyogo 659-8502, Japan

E-mail: n.araki@ashiya-hosp.com

Key words: rectotumoral fistula, sacral chordoma, carbon ion radiotherapy, postradiation sarcoma the standard cure management for sacral chordoma, whereas radiotherapy has been used for radical treatment in inoperable patients. However, carbon ion radiotherapy (CIRT) has recently emerged as a promising treatment for unresectable sacral chordoma in Japan (3). Heavy ions, such as carbon ions, have less lateral scattering than that of photons, which leads to further improvement in the dose distribution to the target area (4). A distinguishing feature of carbon ions versus photons is the release of low-level radiation along their travel paths except for the maximum energy at the end of their range (Bragg peak) (4). Thus, CIRT can selectively irradiate tumors by sparing the surrounding normal tissues $(4,5)$. Furthermore, the relative biological effectiveness (RBE) in the tumor region is higher for heavy ions than for photons (5). Previous reports have demonstrated that patients who received marginal or intralesional excision of sacral chordoma often have worse prognoses, and adequate margins are only achieved in roughly $50 \%$ of cases. In addition, even for widely resected sacral chordoma, the 5 -year local control rate has been found to be $80 \%$ (6-10). On the other hand, Imai et al reported a 5-year local control rate of sacral chordoma treated by CIRT of $88 \%$, although $>80 \%$ of tumors in the study were located higher than the S2 level (3). Additionally, CIRT has fewer short-term complications than does surgical resection for sacral chordoma $(3,11)$. However, when sacral chordoma is close to the intestine, spacers are often placed between the tumor and the intestine to reduce the risk of gastrointestinal complications (4). In Japan, CIRT has increasingly been used for treatment of sacral chordoma. Here we present two cases of rectotumoral fistula formation that occurred $>5$ years after CIRT for sacral chordoma. We obtained written informed consent from the two patients.

\section{Case report}

Case 1. A 73-year-old man was referred to our outpatient clinic for sacral tumor detected on magnetic resonance imaging 

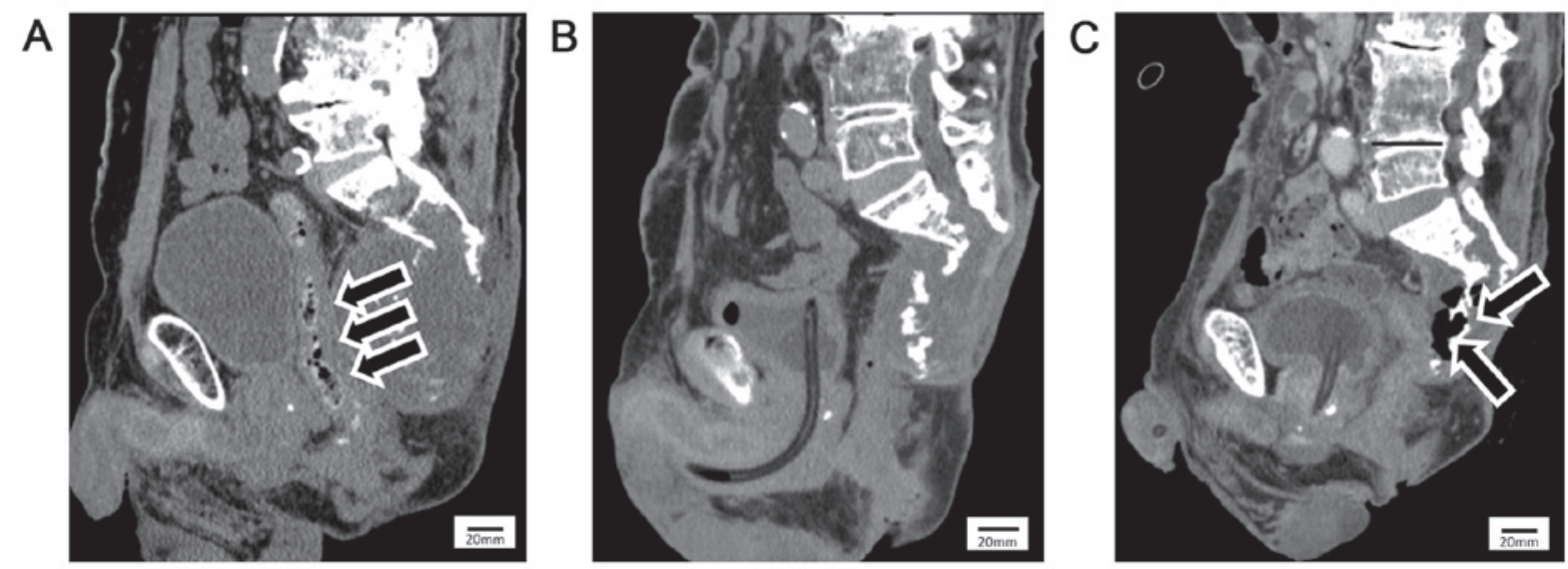

Figure 1. Sagittal computed tomographic images in Case 1. (A) Image prior to CIRT: The tumor is close to the rectum (arrows). (B) Image 6 years following CIRT: The tumor has shrunk markedly and is partially ossified. (C) Image 7 years following CIRT: A rectotumoral fistula has formed and emphysema can be seen inside the tumor (arrows). CIRT, carbon ion radiotherapy.
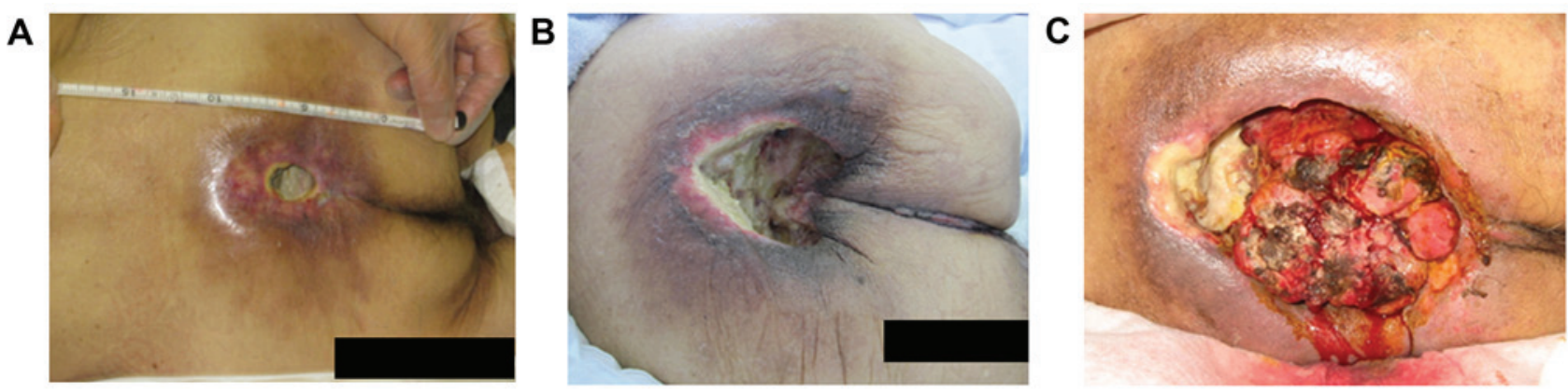

Figure 2. Images of the hip in Case 1. (A) Image of the patient at time of admission to the hospital: Skin flare is centrally ulcerated around the sacrum. (B) Image following several surgeries: Large ulceration without wound healing. (C) Image several days prior to mortality: Larger ulceration prior and a tumor inside the dimple with continuous bleeding are presented.

(MRI). The tumor was pathologically confirmed by biopsy as a chordoma. Since the tumor invaded the upper level of the sacrum (S1-5) and resection of the tumor would cause severe impairments, the patient decided to receive CIRT instead of surgery.

Since the tumor was contiguous with the rectum (Fig. 1A), we tried to place a spacer between the tumor and rectum to prevent radiation enterocolitis. However, we could not place the spacer because of adhesion of the tumor to the surroundings, so we performed a colostomy before CIRT. We performed CIRT at a total dose of 70.4 Gy (RBE) in 16 fractions. Colostomy closure was not performed because of a neurogenic rectal disturbance that occurred before CIRT. The tumor shrank markedly and partially ossified 6 years after CIRT, as shown by computed tomography (CT) (Fig. 1B) and MRI. Seven years after CIRT, the patient visited a general practitioner because of a high-grade fever and worsening sacral pain. A colonoscopy revealed rectotumoral fistula formation. After antibiotic treatment failed to reduce the high fever, he was referred to our outpatient clinic. Contrast-enhanced CT showed a rectotumoral fistula and emphysema inside the tumor (Fig. 1C). Physical examination showed skin flare that was centrally ulcerated around the sacrum (Fig. 2A), and a tumor was observed in the dimple of ulceration. A blood test showed that his white blood cell (WBC) count and C-reactive protein (CRP) were elevated: WBC, $1.12 \times 10^{4} / \mu 1 ; \mathrm{CRP}, 13.1 \mathrm{mg} / \mathrm{dl}$. He was diagnosed as having sepsis from the rectotumoral fistula with tumor recurrence and received continuous intravenous antibiotic treatment. Resection of the fistula and residual rectum with anal closure and necrotic sacral bone excision were performed to control the infection 1 month after admission. During the procedures, a tumor biopsy was performed. However, after the operation, the ulceration enlarged and the tumor kept growing (Fig. 2B). Pathological examination revealed that most components appeared as undifferentiated pleomorphic sarcoma and some parts still resembled a chordoma, however, brachyury staining, which is diagnostic marker for chordoma, was negative. The tumor showed no identifiable line of differentiation based on the immunohistochemical negativity for pan-cytokeratin, epithelial membrane antigen (EMA), desmin, $\alpha$-smooth muscle actin ( $\alpha$-SMA), S100, and CD34; all of which were negative in the morphologically chordoma-like areas as well. The specimen was considered as either a post-CIRT sarcoma or a recurrent dedifferentiated chordoma (Fig. 3). Despite trans-arterial embolization (TAE) and chemical debridement using Mohs paste, the tumor kept growing and bleeding. Finally, he died 9 years after CIRT because of bleeding from the tumor (Fig. 2C).

Case 2. A 55-year-old man was referred to our outpatient clinic for an expanding tumor, which was previously diagnosed as a benign tumor by biopsy when he received surgery for rectal cancer 7 years ago. We performed a biopsy again, and the specimen was pathologically confirmed as chordoma. 
A

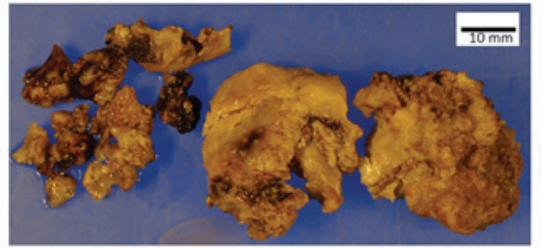

B

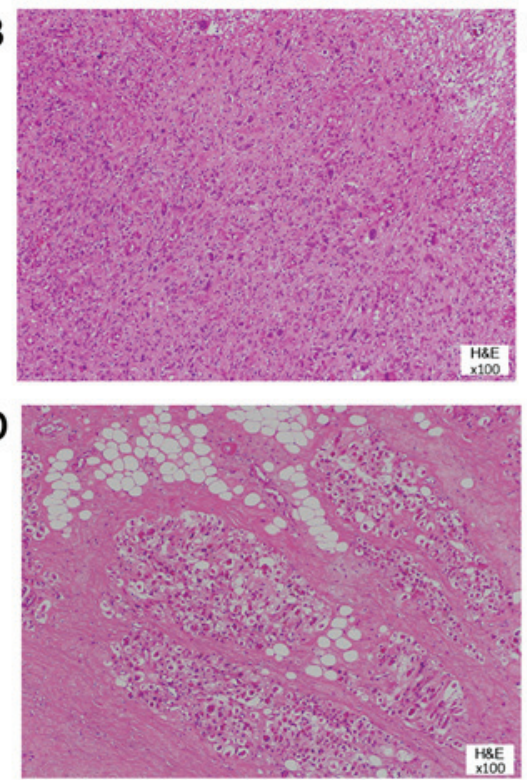

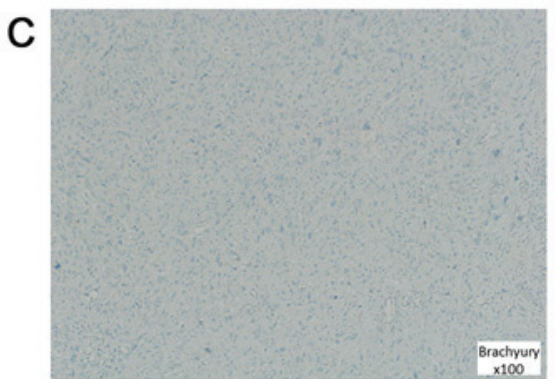

E

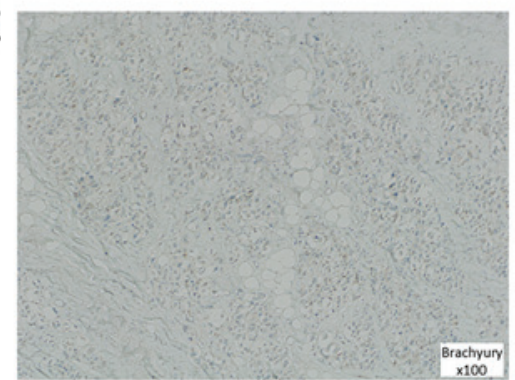

Figure 3. Biopsy of Case 1. (A) Tumors biopsied in the dimple. Pathological examination of high grade sarcoma lesion by (B) hematoxylin and eosin (H\&E) and (C) brachyry staining. Pathological examination of chordoma-like appearance lesion by (D) H\&E and (E) brachyry staining. Magnification, x100.
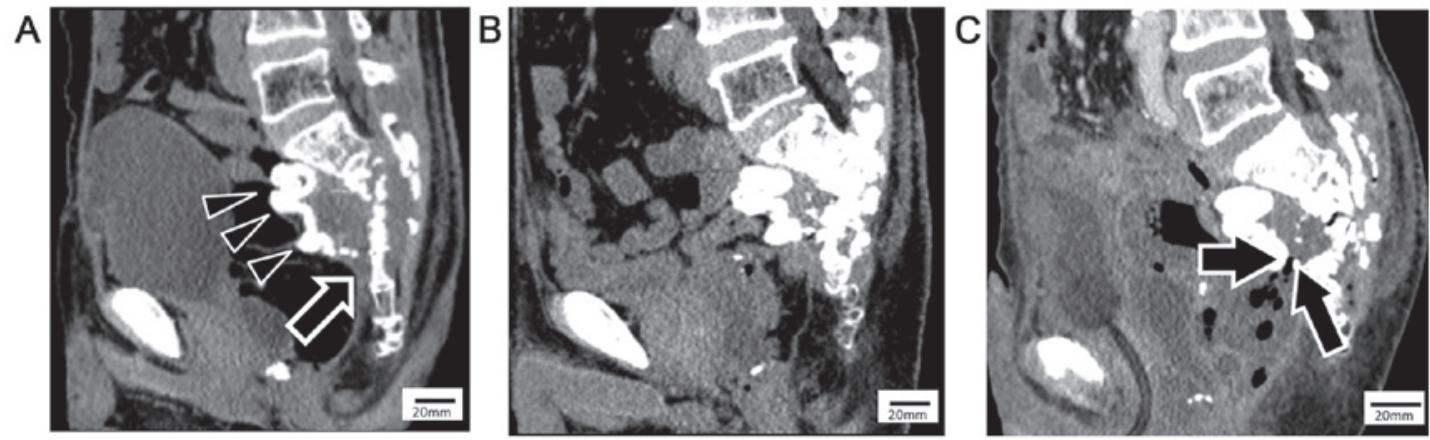

Figure 4. Sagittal computed tomographic images in Case 2. (A) Image prior to CIRT: The placed spacer placed shown (triangles); however, the tumor in front of the coccyx is uncovered (arrows). (B) Image 8 years following CIRT: The tumor has shrunk markedly and become partially ossified. (C) Image 10 years following CIRT: A rectotumoral fistula has formed at the site uncovered by the spacer (arrows) and emphysema may be seen inside the tumor. CIRT, carbon ion radiotherapy.

Surgical resection had a risk of inducing severe neurological deficit because the tumor invaded the upper level of the sacrum (S1-5). Thus, he rejected the surgery and accepted CIRT instead. The total dose was $70.4 \mathrm{GyE}$ in 16 fractions. A $15 \times 20-\mathrm{cm}$ polytetrafluoroethylene spacer was placed between the rectum and tumor before CIRT to prevent radiation enterocolitis. The tumor in front of the coccyx could not be covered because of adhesion of the rectal surroundings caused by previous surgery (Fig. 4A). The tumor had shrunk markedly and become partially ossified 8 years after CIRT (Fig. 4B). However, follow-up MRI and CT revealed tumor regrowth and lung metastasis 10 years after CIRT. Surgery had not been performed because the tumor had invaded the pelvic structures, and radiotherapy could not be delivered because of the previous CIRT. He visited our hospital because of a high fever 1 month after diagnosis of the tumor regrowth. A blood test revealed high WBC count $\left(1.12 \times 10^{4} / \mu \mathrm{l}\right)$ and CRP $(20.0 \mathrm{mg} / \mathrm{dl})$, and a blood culture was positive for proteus vulgaris. Contrastenhanced CT revealed that the rectotumoral fistula at the site of the spacer was uncovered and emphysema was inside the tumor (Fig. 4C). He was diagnosed as having sepsis from the rectotumoral fistula that was difficult to remove and received intravenous antibiotic treatment. He died of uncontrolled sepsis 7 months after admission despite continuous antibiotic treatment.

\section{Discussion}

There are few reports about complications of CIRT for sacral chordoma. Neuropathic pain (15-16\%) and skin toxicity (5-22\%) have been reported $(3,11)$. Imai et al reported 188 cases analysis of CIRT for unresectable sacral chordoma. In that study, the median follow-up period was 62 month (6.8-147.5) and $70 \%$ of patients were followed for over 5 years or until death. The 5-year local control rates were $77.2 \%(3,12)$. The local recurrence rate is not high. Among the locally recurrent cases there are no previous report about fistula formation. Thus, it is quite rare complication, and to our best knowledge, this is the first report of rectotumoral fistula formation after CIRT for sacral chordoma. 
We considered two possible explanations for the fistula formation: First, radiation enterocolitis after CIRT might have caused formation of the fistula over a long period of time (13). Wallner et al reported that $8(0.3 \%)$ of 2464 patients developed a radiation-related fistula after prostate brachytherapy (13). The average volume of the rectum receiving $100 \%$ of the prescription dose was moderately higher for the fistula patients than for the non-fistula patients. Time to fistulation ranged from 23 to 66 months (median, 25 months) (13). Thus, irradiation of the gastrointestinal tract around the tumor has a risk for fistula formation. Although both of our patients were operated on for spacer protection, coverage of the rectum was not achieved, which led to fistulation. Therefore, it is important to be aware that when sufficient protection during CIRT cannot be provided, careful follow-up to monitor potential fistulation is necessary. Second, tumor regrowth can compress and invade the rectum and enforce fistula formation. Imai et al reported that the local recurrence rate for 5 years was $22.8 \%$ (12). However, the long-term recurrence rate remains unknown. In our cases, the patients experienced tumor regrowth 7 years (Case 1) and 10 years (Case 2 ) after CIRT and increased rapidly. Late tumor regrowth might affect the long latency of fistula formation.

We confirmed dedifferentiated morphology similar to undifferentiated pleomorphic sarcoma by biopsy 7 years after CIRT in Case 1. Huvos et al proposed the following criteria for diagnosis of post-radiation sarcoma: i) The patient received irradiation, ii) the neoplasm occurred in the radiation field, iii) a latent period of some years had elapsed, and iv) there was histological or roentgenographic evidence for the pre-existing osseous condition, if present, in addition to microscopic proof of a sarcoma (14). The risk of post-radiation sarcoma has been found to be $0.06 \%$ at a mean latency of 15 years (3-50 years) (15). It is difficult to determine whether our cases involved dedifferentiated chordomas or post-CIRT sarcomas. However, according to Huvos' criteria, we concluded that it was post-CIRT sarcoma.

The limitation of our study is little pathological evidences to show sarcoma formation from chorodoma. In Case 1, unfortunately, the pathology preparation slide before CIRT was lost. However, needle biopsy under CT guide was performed. The pathological report was slow growing chordoma. We confirmed the diagnosis as chordoma with the result of this needle biopsy and the typical radiographic feature of MRI and $\mathrm{CT}$. The radiographic feature represent no evidence of high grade sarcoma before CIRT. After CIRT, some parts of tumor still resembled a chorodma. However, brachyury staining was negative. In Case 2, we also could not find the pathological preparation slide nor paraffin block of initial open biopsy specimen in case 2. However, this case was irradiated in National Institute of Radiological Sciences in Chiba, where all cases were pathologically and radiographically reviewed by the board members. This case was also centrally reviewed and diagnosed as typical chordoma. We did not perform biopsy for recurrent tumor because of the sepsis and malnourishment.

It is important to prevent fistula formation at the tumor because treatments of the wound within the radiation area of CIRT are very difficult. Unfortunately, when a tumor is found that shows regrowth, resection of the tumor including the residual rectum should be considered.

In conclusion, we experienced two cases of rectotumoral fistula formation that occurred $>5$ years after CIRT. It is important to be aware of the possibility of rectal complications and to investigate rectal conditions after CIRT during long-term follow-up. If rectal complications are revealed, resection of the rectum should be considered. Additionally, tumor regrowth can worsen rectal complications.

\section{Acknowledgements}

Not applicable.

\section{Funding}

No funding was received.

\section{Availability of data and materials}

The datasets generated and/or analyzed during the current study are not publicly available to maintain the privacy of the patients but are available from the corresponding author on reasonable request.

\section{Authors' contributions}

YU collected the patient data and was a principal contributor to the writing of the manuscript. NA contributed to the design and conception of the present study, and corrected the manuscript. HO additionally contributed to the design and conception of the present study, and reviewed the manuscript. SN and EK performed the histological examination of chordoma specimens. RI treated the patient by carbon ion radiotherapy in Case 2 and contributed to data collection. YD and TO treated the patient by carbon ion radiotherapy in Case 1 and contributed to data collection. NN contributed to the data collection. All authors contributed to the interpretation, discussion and critical review of the manuscript. All authors read and approved the final manuscript.

\section{Ethics approval and consent to participate}

The present study was approved by the Osaka International Cancer Institutional Review Board (Osaka, Japan).

\section{Patient consent for publication}

The patients provided informed consent regarding medical information and the publication of the present study.

\section{Competing interests}

The authors declare that they have no competing interests.

\section{References}

1. Yu E, Koffer PP, DiPetrillo TA and Kinsella TJ: Incidence, treatment, and survival patterns for sacral chordoma in the United States, 1974-2011. Front Oncol 6: 203, 2016.

2. Chugh R, Tawbi H, Lucas DR, Biermann JS, Schuetze SM and Baker LH: Chordoma: The nonsarcoma primary bone tumor. Oncologist 12: 1344-1350, 2007.

3. Imai R, Kamada T, Sugahara S, Tsuji H and Tsujii H: Carbon ion radiotherapy for sacral chordoma. Br J Radiol 84: S48-S54, 2011. 
4. Lorenzo C, Andrea P, Barbara V, Denis P, Rosaria FM, Piero F, Viviana V, Alberto I, Mario C, Brugnatelli S, et al: Surgical spacer placement prior carbon ion radiotherapy (CIRT): An effective feasible strategy to improve the treatment for sacral chordoma. World J Surg Oncol 14: 211, 2016.

5. Kamada T, Tsujii H, Blakely EA, Debus J, De Neve W, Durante M, Jäkel O, Mayer R, Orecchia R, Pötter R, et al: Carbon ion radiotherapy in Japan: An assessment of 20 years of clinical experience. Lancet Oncol 16: e93-e100, 2015.

6. Varga PP, Szövérfi Z, Fisher CG, Boriani S, Gokaslan ZL, Dekutoski MB, Chou D, Quraishi NA, Reynolds JJ, Luzzati A, et al: Surgical treatment of sacral chordoma: Prognostic variables for local recurrence and overall survival. Eur Spine J 24: 1092-1101, 2015

7. Radaelli S, Stacchiotti S, Ruggieri P, Donati D, Casali PG, Palmerini E, Collini P, Gambarotti M, Porcu L, Boriani S, et al: Sacral chordoma: Long-term outcome of a large series of patients surgically treated at two reference centers. Spine 41: 1049-1057, 2016.

8. Yang Y, Niu X, Li Y, Liu W and Xu H: Recurrence and survival factors analysis of 171 cases of sacral chordoma in a single institute. Eur Spine J 26: 1910-1916, 2017.

9. Stacchiotti S and Sommer J; Chordoma Global Consensus Group: Building a global consensus approach to chordoma: A position paper from the medical and patient community. Lancet Oncol 16: e71-e83, 2015.

10. Asavamongkolkul A and Waikakul S: Wide resection of sacral chordoma via a posterior approach. Int Orthop 36: 607-612, 2012.
11. Demizu Y, Jin D, Sulaiman NS, Nagano F, Terashima K, Tokumaru S, Akagi T, Fujii O, Daimon T, Sasaki R, et al: Particle therapy using protons or carbon ions for unresectable or incompletely resected bone and soft tissue sarcomas of the pelvis. Int $\mathrm{J}$ Radiat Oncol Biol Phys 98: 367-374, 2017.

12. Imai R, Kamada T, Araki N, Abe S, Iwamoto Y, Ozaki T, Kanehira C, Kaya M, Takahashi K, Chuman H, et al; Working Group for Bone and Soft Tissue Sarcomas: Carbon ion radiation therapy for unresectable sacral chordoma: An analysis of 188 cases. Int J Radiat Oncol Biol Phys 95: 322-327, 2016.

13. Wallner K, Sutlief S, Bergsagel C and Merrick GS: Severe rectal complications after prostate brachytherapy. Radiother Oncol 114: 272-275, 2015.

14. Huvos AG, Woodard HQ, Cahan WG, Higinbotham NL, Stewart FW, Butler A and Bretsky SS: Postradiation osteogenic sarcoma of bone and soft tissues. A clinicopathologic study of 66 patients. Cancer 55: 1244-1255, 1985.

15. Mavrogenis AF, Pala E, Guerra G and Ruggieri P: Post-radiation sarcomas. Clinical outcome of 52 Patients. J Surg Oncol 105: 570-576, 2012.

This work is licensed under a Creative Commons Attribution-NonCommercial-NoDerivatives 4.0 International (CC BY-NC-ND 4.0) License. 